Résumés des conférences et travaux

\title{
Langue et littérature hébraïques dans l'Occident médiéval et moderne
}

Jean-Pierre Rothschild

\section{OpenEdition}

Journals

Édition électronique

URL : https://journals.openedition.org/ashp/3487

DOI : $10.4000 /$ ashp.3487

ISSN : 1969-6310

Éditeur

Publications de l'École Pratique des Hautes Études

Édition imprimée

Date de publication : 1 septembre 2020

Pagination : 37-43

ISSN : 0766-0677

Référence électronique

Jean-Pierre Rothschild, "Langue et littérature hébraïques dans l'Occident médiéval et moderne »,

Annuaire de l'École pratique des hautes études (EPHE), Section des sciences historiques et philologiques

[En ligne], 151 | 2020, mis en ligne le 09 juillet 2020, consulté le 06 juillet 2021. URL : http://

journals.openedition.org/ashp/3487 ; DOI : https://doi.org/10.4000/ashp.3487 


\title{
LANGUE ET LITTÉRATURE HÉBRAÏQUES DANS L'OCCIDENT MÉDIÉVAL ET MODERNE
}

\author{
Directeur d'études : M. Jean-Pierre RothschILD
}

Programme de l'année 2018-2019 : I. Joseph ibn Shem Tov et l'Épître sur la conjonction avec l'intellect agent d'Averroès. - II. Juda de Rome, commentaire du Qaddiš et de la Qedušah et autres inédits.

Actif à Ségovie dans la décennie 1440 et au début de la suivante, le philosophe juif castillan Joseph ben Shem Tov occupe une position singulière ${ }^{1}$ : fils d'un kabbaliste qui fut adversaire déclaré de la philosophie, frère et père de deux philosophes adhérant à la tradition de lecture rationaliste ${ }^{2}$ de la pensée de Maïmonide, il est luimême à la fois un adversaire du rationalisme en philosophie, un excellent connaisseur des sources du savoir philosophique accessibles dans l'Espagne juive du $\mathrm{XV}^{\mathrm{e}}$ siècle et le chef d'un collège (yešibah) où l'on étudie la philosophie ${ }^{3}$. C'est en cette qualité, sans doute, qu'il rédigea, à la fin de la période d'activité que nous lui connaissons, une série de commentaires, presque tous inédits à l'heure qu'il est ${ }^{4}$, de textes portant sur la question classique mais centrale et ô combien difficile de la manière dont l'homme accède à l'intellection : commentaires long et court sur la Lettre d'Averroès sur la possibilité de la conjonction avec l'intellect agent, commentaires de son commentaire sur celle d'Alexandre d'Aphrodise sur le même sujet, du traitement de l'intellect par Moïse de Narbonne dans son commentaire aux Intentions des philosophes d'al-Ghazâlî, du traitement par Maïmonide du thème de l'unité de l'intellect et de son sujet (Guide des égarés I, 68), commentaire sur l'explication de l'union de l'intellection, de l'intelligent et de l'intelligible selon Averroès et commentaire partiel sur le commentaire d'Averroès au 1. III du De anima d'Aristote. L'ensemble de ces textes

1. Réinterprétée, contre plusieurs jugements inexacts (de «philosophie en demi-teinte») portés au $\mathrm{Xx}^{\mathrm{e}}$ siècle, par J.-P. Rothschild, «Le dessein philosophique de Joseph Ibn Shem Tob (Espagne, flor. 1442-1455) », Revue des études juives, 162, 1-2 (janv.-juin 2003), p. 97-122. Cependant, des évaluations perspicaces avaient été faites bien plus tôt par Moritz Steinschneider, « Josef b. Schemtob’s Commentar zu Averroes' Größer Abhandlung über die Möglichkeit der Conjunction », Monatsschrift für Geschichte und Wissenschaft des Judent(h)ums, 32 (1883), p. 459-479, 514-521 (460) puis par Israel Zinberg, A History of Jewish Literature, t. III. The Struggle of Mysticism and Tradition Against Philosophical Rationalism, Bernard Martin (trad. et éd.), Cleveland, Londres, The Press of Case Western Reserve University, 1973, p. 244-254.

2. Rappelons que nous entendons par rationalisme, dans ce contexte, la position qui consiste à considérer que la Torah n'enseigne, sous un autre mode, rien d'autre que ce qu'enseigne la philosophie.

3. Yoel Marciano, Sages of Spain in the Eye of the Storm. Jewish Scholars of Late Medieval Spain (en hébreu), Jérusalem, The Bialik Institute, 2019, p. 129-130.

4. La seule exception est une édition du commentaire court à l'épître d'Averroès sur la possibilité de la conjonction, procurée par S. Regev, l'auteur de l'unique thèse consacrée à ce jour à Joseph ben Shem Tov. Mais il s'est contenté d'une transcription du texte, qui comporte plusieurs passages non lus, en s'abstenant de toute annotation et de toute présentation doctrinale de ce texte difficile. 
fut écrit dans une période étrangement concentrée, à l'automne 1454, comme en font foi les colophons d'auteur ${ }^{5}$.

Nous avions envisagé dans notre article de 2003 que cette hâte pût s'expliquer par le sentiment, de la part de Joseph ben Shem Tov, d'avoir fait une découverte majeure, peut-être celle du moyen de justifier philosophiquement la possibilité pour les simples fidèles de parvenir aux plus hautes intellections sans passer par le long système de l'apprentissage des sciences que prévoit le rationalisme maïmonidien en réservant ainsi l'accès à la félicité la plus élevée à une élite de savants, à l'exclusion du plus grand nombre des observants peu instruits, en quoi son propre père avait vu une erreur des philosophes aux lourdes conséquences sociales. Le retour que nous effectuons présentement sur ces textes ne permet pas de soutenir une telle hypothèse. Les commentaires de Joseph ben Shem Tov comportent une large part d'explications techniques, souvent suivies d'objections personnelles contre les positions des philosophes. Loin de trouver chez eux les moyens de frayer une voie nouvelle, il identifie et critique leurs positions contraires aux enseignements toraïques. Comment, donc, expliquer la hâte rédactionnelle dont témoigne cet ensemble de textes? On peut avancer trois raisons, d'ailleurs hétérogènes : l'unité du sujet traité, chaque texte, du reste, renvoyant aux autres; l'âge

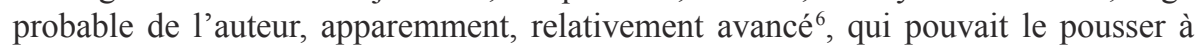
vouloir terminer son traitement d'une question essentielle; enfin, il semble probable que Joseph b. Shem Tov dictait ses textes : dans le prologue du 'Eyn ha-qôrê', écrit entre 1442 et 1455, il s'excuse du désordre de son texte sur ce qu'il est aveugle; quand bien même cette cécité n'aurait été que temporaire, le prologue en question ne signale qu'un changement induit, en fait d'ordre, sans référence à d'autres modifications de ses conditions de travail qu'aurait amenées le truchement d'un scribe et d'un relecteur s'il avait été inhabituel; dans l'ensemble de ses textes, la syntaxe apparaît en maints endroits décousue; familier de la cour des rois de Castille Jean II et Henri IV en qualité de médecin et de réviseur des comptes, Joseph occupait une position sociale compatible avec le recours à des secrétaires; plus largement, l'étonnante prolixité d'autres auteurs juifs de la péninsule Ibérique au $\mathrm{XV}^{\mathrm{e}}$ siècle, eux aussi investis de responsabilités curiales et, en outre, de charges communautaires, comme Hasdaï Crescas à la fin du XIV ${ }^{\mathrm{e}}$ et au début du XV $\mathrm{XV}^{\mathrm{e}}$ siècle et Isaac Abrabanel à la fin du XV $\mathrm{XV}^{\mathrm{e}}$, ne paraît explicable que moyennant cette facilitation ${ }^{7}$.

Nous n'avons pas, cette année, abordé directement le commentaire long sur la Lettre d'Averroès, comme l'indiquait notre titre, en raison de sa longueur; mais lu en partie et parcouru pour le reste le Commentaire sur le commentaire d'Averroès à la Lettre d'Alexandre d'Aphrodise sur la possibilité de la conjonction de l'intellect

5. J.-P. Rothschild, « Le dessein philosophique de Joseph Ibn Shem Tob », p. 107-108.

6. Y. Marciano, Sages of Spain, p. 129, n. 277, propose avec un bon argument d'avancer vers 1385 la naissance de Joseph ben Shem Tov, habituellement située sans raison particulière vers 1400 .

7. Je n'ai pas connaissance que cette hypothèse ait été signalée, démentie ou documentée quelque part; il y aurait à tenter une étude des conditions de travail de ces intellectuels hommes de cour. — Quant à ce que la syntaxe du seul Joseph paraît se ressentir de cette pratique supposée, il suffit de noter que les deux autres auteurs allégués sont en général lus d'après des éditions imprimées dont on peut supposer que les éditeurs ont procédé à une mise au point des textes; la comparaison avec leurs manuscrits, comme aussi celle du seul texte imprimé de Joseph avec l'unique manuscrit qui en subsiste, seraient à faire. Aussi bien, d'autres auteurs auraient-ils pu dès l'état manuscrit se soucier d'une révision à laquelle Joseph n'aurait pas procédé ou fait procéder. 
humain avec l'intellect agent, d'argument proche, qui d'ailleurs s'y réfère et que nous pouvions espérer lire en une ou deux années de conférences. Ce texte est connu par trois manuscrits, New York, Jewish Theological Seminary 2341, f. 250-260; Oxford, Bodleian Library, Oppenheim Add. 4 21 (catalogue Neubauer : $n^{\circ} 1353$ ), f. 19-41; Paris, Bibliothèque nationale de France, hébreu 1004, f. 121v-144. Le second est à peu près illisible, l'encre ayant corrodé le papier, un phénomène fréquent parmi les manuscrits hébreux du $\mathrm{Xv}^{\mathrm{e}}$ siècle d'origine espagnole. Nous avons utilisé les deux autres, qui se complètent assez bien. Le ms. de New York, un recueil de près de quatre cents feuillets de textes de Joseph b. Shem Tov, puis de son fils Shem Tov, copié d'une main espagnole de la deuxième moitié $\mathrm{du} \mathrm{Xv}^{\mathrm{e}}$ siècle, est par moments d'une grande difficulté de lecture et n'est pas dépourvu d'omissions par saut du même au même (ce qui exclut qu'on puisse penser qu'il résulte directement d'une dictée d'auteur). Le manuscrit de Paris, au contenu philosophique particulièrement intéressant (à noter, outre divers textes de Joseph $\mathrm{b}$. Shem Tov et de contemporains espagnols, Abraham Bibago, Isaac Abrabanel et Isaac Ibn Latif, des traductions en hébreu de textes de Robert Grosseteste [f. 106-121v] dont il est l'un des rares témoins), de lecture aisée, comporte lui aussi des omissions qui ne coïncident pas avec celles du manuscrit newyorkais, davantage de fautes de lecture et assez souvent des blancs laissés par le copiste quand il ne déchiffrait pas son modèle; le recours aux deux fournit un texte raisonnablement lisible. La méthode du commentaire est claire : dans le manuscrit de Paris, de longs passages d'Averroès sont cités intégralement, sous l'entrée « Averroès dit ", puis font l'objet, sous l'entrée « commentaire », d'une longue périphrase littérale mais aussi explicative qui cite à nouveau le texte commenté phrase par phrase, le reproduisant ainsi, à nouveau, en entier (le témoin de New York abrège sévèrement et réduit à presque rien tant les longs passages que les phrases reprises); dans le présent commentaire, à la différence de ce qui s'observe ailleurs, la critique du texte commenté n'apparaît qu'une fois (héb. 1004, f. 128-129v), sous l'intitulé : "Joseph dit ». Comme il l'expose à la fin de son introduction à la Lettre d'Averroès, il existe trois explications philosophiques de la perfection de l'âme humaine : selon Alexandre d'Aphrodise, une entéléchie (hakhanah, « disposition ») dans l'intellect humain, ou matériel (hylique), unit les formes intelligibles à la faculté imaginative; selon Thémistius, ce rôle est dévolu à une substance (un intellect); Averroès l'admet mais moyennant que soit liée au corps de l'homme une faculté qui lui permet la connaissance intellectuelle, dont n'ont pas besoin les intellects séparés. C'est ici la position d'Alexandre qui est analysée à travers le commentaire qu'en a donné Averroès. Il s'agit d'abord de définir l'intellect hylique comme une puissance, séparée du corps (sinon de l'imagination) de l'homme, de recevoir les intelligibles en acte, à la manière de la cire d'une tablette en mesure de recevoir l'écriture, laquelle cependant ne s'inscrit pas dans le bois de la tablette mais seulement sur la cire qui y est déposée. Cette définition fait difficulté; en effet, ce qui passe de la puissance à l'acte est soumis au changement, donc à la génération et à la corruption, relève du possible et non du nécessaire; or, cela se heurte à trois objections : il serait « honteux» (entendre : incompatible avec leurs définitions) que la nature d'un intellect fût soumise à la génération et à la corruption et, par suite, qu'en fussent atteintes les formes intelligibles qui se trouvent en lui; d'autre part, comment cela se pourrait-il, alors que l'intellect virtuel-disposition dont on parle aurait 
un caractère séparé? Enfin, c'est contraire à l'autorité d'Aristote qui a estimé que l'intellect matériel était éternel. La réponse à ces objections consiste à dire que l'intellect matériel serait corruptible s'il était une faculté séparée dans chacun des individus de l'espèce humaine, mais qu'en vérité il est une faculté unique séparée, reliée seulement aux individus, de la même manière que l'espèce humaine, qui est une, se trouve en chacun d'eux; cependant, l'espèce, hors des individus, est en puissance, en acte seulement en eux; au contraire, cet intellect est en acte hors d'eux, sans substrat, tandis qu'en eux, elle est en puissance. Ainsi pourra-t-on comprendre que les formes intellectuelles soient éternelles bien que les individus humains qui se les représentent soient mortels. Averroès montre en effet dans le chapitre suivant de son commentaire que, par suite, les intelligibles présents à cette faculté échappent eux aussi, en euxmêmes, à la génération et à la corruption, aussi bien qu'à la multiplicité, encore qu'ils soient créés en chaque individu. C'est là la solution propre d'Averroès élaborée pour sortir de la difficulté exposée plus haut, en opposition aux thèses d'Alexandre (l'intellect matériel, en tant que disposition, est périssable) et de Thémistius (c'est une substance distincte). À ce stade, Joseph b. Shem Tov expose ses propres objections. Il dresse d'abord une liste de passages dans lesquels Averroès a traité de la même question en tenant des positions contradictoires. Il avance ensuite une série de difficultés : 1) la faculté dont il parle serait à la fois une et grosse de multiples possibles, ce qui est contradictoire; 2) une espèce ne saurait être en acte en dehors des individus qui la composent; 3) quoique supposée séparée, si tous les individus humains périssaient, elle n'aurait nulle existence; 4) les intelligibles ne sauraient exister hors de tout intellect, il faudrait donc qu'ils soient toujours présents dans quelque intellect, d'où, que l'intellect hylique ne serait pas une simple disposition, que l'homme les connaîtrait sans apprentissage, et que l'intellect hylique ne différerait pas d'un intellect séparé (ou : serait inutile). Joseph ben Shem Tov se livre ensuite à une critique de la théorie de l'éternité des espèces, telle que la soutient aussi Aristote, avant de reprendre le fil du commentaire d'Averroès.

Resterait, pour conclure provisoirement, à s'interroger sur sa démarche. Nous l'avions déjà fait dans notre article de 2003 mais, depuis lors, nous avons appris deux choses : par $\mathrm{M}^{\mathrm{me}}$ Colette Sirat puis par M. Yoel Marciano, qu'il est au nombre de ceux qui tinrent en Espagne une « yešibah de sciences (philosophiques) » et, par nos propres lectures de ses commentaires philosophiques, qu'il n'en tire pas une voie nouvelle et rapide vers la félicité spirituelle. Nous voudrions d'abord mettre en garde contre la formule de «yešibah philosophique » et ce qu'elle implique : qu'il y aurait des écoles de philosophie comme il y a des écoles de Talmud, ou bien, que dans certaines écoles, d'abord destinées à l'apprentissage du Talmud, se pratiquait aussi celui de la philosophie; dans les deux cas, la philosophie aurait été une option. Une telle vue ne tient pas compte des âpres querelles autour de l'âge des études de philosophie, pourtant toujours rappelées en prologue à ce type de recherches : il n'y a matière à querelles publiques que parce que toute la jeunesse lettrée se trouve exposée à la philosophie, à un moment dont l'enjeu de ces querelles est la fixation. Elle n'explique pas non plus la familiarité avec un vocabulaire et des références philosophiques manifestée par bien des auteurs critiques de la philosophie, qui ne sauraient tous avoir été des transfuges ou les bénéficiaires par exception d'une formation double : qu'il suffise de nommer Hasdaï Crescas, Shem Tov Ibn Shem Tov, Joseph Ibn Shoshan, 
Joseph Albo, Joseph Ya'abeș, Isaac Abrabanel même. Les positions discordantes visà-vis de la philosophie manifestées dans la famille Ibn Shem Tov supposent elles aussi une formation commune selon un modèle imposé auquel même un adversaire déclaré des philosophes comme Shem Tov Ibn Shem Tov n'avait pas songé à soustraire ses fils. Que la philosophie ait ainsi été une composante indispensable de la formation des lettrés peut s'expliquer par son caractère d'horizon intellectuel indépassable du moment; par la nécessité pratique de faire bonne figure dans les cours, devant les clercs et, dans ce Moyen Âge tardif, aussi devant de nombreux laïcs instruits, fût-ce en langues vernaculaires; et par celle de pouvoir résister ou répondre aux discours convertisseurs des clercs, imposés aux juifs par les autorités ${ }^{8}$ ou imprégnant les rapports intercommunautaires courants ${ }^{9}$. Enfin, le modèle intellectuel prestigieux de l'université chrétienne ne pouvait manquer d'exercer son emprise.

Dès lors, c'est de l'intérieur de ce cadre obligé qu'un homme comme Joseph b. Shem Tov a développé sa critique de la philosophie. Nous l'avons déjà montré à propos de sa critique de Maïmonide dans le 'Eyn ha-qore', dans les Mélanges Charles Touati, parus en 2001. Sans préjuger de l'ensemble des critiques qu'il formule dans le groupe de commentaires sur les principaux textes philosophiques consacrés aux processus de l'intellection humaine, dont nous reprenons seulement l'étude, nous pouvons du moins observer que, dans le cadre de son commentaire à celui d'Averroès sur la Lettre d'Alexandre d'Aphrodise, il met en cause, outre des inconséquences du Commentateur, la notion aristotélicienne de l'éternité des espèces et des intelligibles, peut-être dans l'idée qu'elle contredit la notion de création.

Juda b. Mosheh b. Dani'el Romano, né vers 1292, fut chef d'école, comme en témoigne son cousin Emmanuel de Rome dans ses Maḩbarot : sans doute succéda-t-il à son maître Zerahiyah Hen. De ce que Moïse Rieti le nomme, dans son Miqdaš me 'at, R. Yehudah ha-pilosof, certains ont conclu qu'il avait été considéré comme «le philosophe » juif par excellence. Ce qui est sûr, c'est que les manuscrits de ses œuvres (surtout de ses traductions) furent nombreux en Italie. Il ressort du témoignage d'Emmanuel que Juda vécut et enseigna à Rome. Cela semble confirmé par la connaissance rapide qu'il eut des œuvres récentes de Thomas d'Aquin mais aussi d'auteurs tels qu'Angelo da Camerino, Alexandre le Mineur, Gilles de Rome. Sa personnalité intellectuelle et ses traductions pont été étudiées par Giuseppe (Yosef Barukh) Sermoneta et son élève Caterina Rigo ${ }^{10}$.

8. La pratique en est déjà ancienne : Robert Chazan, Daggers of Faith. 13th-Century Christian Missionizing and Jewish Response, Berkeley, Los Angeles, University of California Press, 1989, p. 38-48; Id., Reassessing Jewish Life in Medieval Europe, Cambridge, Cambridge University Press, 2010, p. 203, 249; Ora Limor, « Polemical Varieties: Religious Disputations in 13th Century Spain », Iberia Judaica, 2 (2010), p. 55-79 (65-66 et n. 33); Paola Tartakoff, Between Christian and Jew. Conversion and Inquisition in the Crown of Aragon, 1250-1391, Philadelphie, University of Pennsylvania Press, 2012 (p. 88-91 sur les sermons donnés par des convertis en Aragon au XIV s.).

9. Gilbert Dahan (intr., éd. et trad.), Ingetus Contardus, Disputatio contra Iudeos, Paris, Les Belles Lettres, 1993, $2^{\text {e }}$ tirage 2012; voir aussi Ram Ben-Shalom, « Between Official and Private Dispute: The Case of Christian Spain and Provence in the Late Middle Ages », AJS Review, 27 (2003), p. 23-71; O. Limor, «Polemical Varieties ».

10. G. Sermoneta, « La dottrina dell'intelletto e la "fede filosofica" di Jehudah e Immanuel Romano », Studi medievali, 6-2 (1965), p. 3-78 et « Jehudàh b. Mošèh b. Dani’el Romano, traducteur de saint 
Sermoneta a remis en contexte la pensée de Juda Romano, rappelant le développement contemporain en Italie, hors des cercles juifs, d'un « averroïsme mystique » qui fit passer la doctrine philosophique de l'union entre l'intellect possible et l'Intellect agent dans le sentiment religieux vécu et lui conféra « la valeur d'un credo ». Il n'y a, pour lui, nulle opposition entre foi et raison ${ }^{11}$ : il convient, dans l'intérêt de la foi, d'être attentif au développement des problématiques intellectuelles de la scolastique, d'où ses travaux de traducteur du latin. Inversement ${ }^{12}$, non seulement l'Écriture (voir son commentaire du récit de la Création), mais encore la liturgie devront être « traduites » en termes philosophiques pour trouver leur justification. Juda Romano, avant de découvrir cette voie, avait, dit-il, cessé de fréquenter la synagogue. Quant à la Cause première, il ne croit pas qu'elle puisse être connue; cependant, l'union à l'Intellect agent, réalisable (comme pour Maïmonide et Avicenne), doit être l'objectif de l'homme de foi, faisant le chemin inverse de celui qui a éloigné l'humanité du jardin d'Éden. L'émanatisme néoplatonicien tiré du Liber de causis que Juda Romano a traduit fait de cette quête un retour à la source ${ }^{13}$.

Parmi ses œuvres originales, on dénombre principalement le commentaire des mots difficiles de Maïmonide ${ }^{14}$; le commentaire de l'CEuvre de la création et l'introduction au commentaire des livres prophétiques ${ }^{15}$; le commentaire du Qaddiš et de la Qedušah, long commentaire philosophique sur les moments capitaux du culte public, inédit; le Sefer Ben porat (Livre du fils fertile), commentaire du compendium de philosophie qui se trouve en tête du Mišneh Torah de Maïmonide ${ }^{16}$.

Le commentaire sur le Qaddiš et la Qedušah est l'œuvre personnelle la plus étendue de Juda Romano et s'annonce comme singulièrement original et central, puisqu'il s'agit d'une interprétation philosophique de deux pièces majeures, y compris la plus connue ${ }^{17}$, la plus fréquente et aussi la plus mystérieuse ${ }^{18}$ de la liturgie

Thomas », dans G. Nahon, C. Touati (éd.), Hommage à Georges Vajda, Louvain, 1980, p. 235-262; C. Rigo, «Yehudah ben Mosheh Romano traduttore degli scolastici latini », Henoch, 17, 1-2 (1995), p. 141-170; Ead., « Egidio Romano nella cultura ebraica: le versioni di Yehudah b. Mosheh Romano », Documenti e studi sulla tradizione filosofica medievale, 5 (1994), p. 397-437; Ead., The Be'urim on the Bible of R. Yehudah Romano: The Philosophical Method which comes out of them, their Sources in the Jewish Philosophy and in the Christian Scholasticism (en hébreu), thèse de l'Université hébraïque de Jérusalem, dactyl., 2 vol., Jérusalem, 1996.

11. G. Sermoneta, « La dottrina », p. 26.

12. Ibid., p. 39.

13. Ibid., p. 57.

14. Éd. et comm. S. Debenedetti Stow, Jehudàh ben Mošèh ben Dani'èl Romano. La chiarificazione in volgare delle «espressioni difficili» ricorrenti nel Mišnèh Toràh di Maimonide. Glossario inedito del XIV secolo, t. I, Rome, 1990, t. II, Turin, 1990.

15. Étudiés dans la thèse de $\mathrm{C}$. Rigo.

16. J.-P. Rothschild, «L'enseignement de la philosophie de Maïmonide selon le Sefer ben porat de R. Juda Romano (Italie, XIV ${ }^{\mathrm{e}}$ siècle) », dans T. Lévy, R. Rached (éd.), Maïmonide, philosophe et savant, (11381204) Louvain, Paris, 2004, p. 433-462.

17. Le Qaddiš est, en matière de judaïsme, « ce qui reste quand on a tout oublié ». Le célèbre testament de Marc Bloch, « Je n'ai pas demandé que sur ma tombe fussent récitées les prières hébraïques dont les cadences, pourtant, accompagnèrent vers leur dernier repos... », y fait évidemment allusion, y compris par harmonie imitative.

18. La dernière étude d'ampleur est à notre connaissance A. Lehnardt, Qaddish. Untersuchungen zur Entstehung und Rezeption eines rabbinischen Gebetes, Tübingen, 2002 (recensé par nous dans la 
juive. Il est conservé au moins dans les manuscrits de Florence, bibl. Laurentienne, plut. I. 22; Parme, bibl. Palatine, De Rossi 129 (Parm. 2834 ; catal. Richler, no 1331); De Rossi 372 (Parm. 2759; Richler, n 1292); De Rossi 1384 (Parm. 3058, Richler $\mathrm{n}^{\mathrm{o}}$ 1295); du Vatican, Biblioteca apostolica Vaticana, Vat. ebr. 90 [copie incomplète, interrompue au milieu du chap. 76]; Vat. ebr. 289; avec des extraits dans Oxford, Bodl. Lib. (cat. Neubauer, $\mathrm{n}^{\circ}$ 2318). L'auteur de l'unique thèse consacrée à Juda Romano, $\mathrm{M}^{\mathrm{me}}$ Caterina Rigo, se promettant toujours d'en publier l'édition critique ainsi que celle de l'ensemble des œuvres de cet auteur, nous ne visons pas nous-même, a priori, à produire une telle édition mais, du moins, un texte de travail. Aussi nous sommesnous contenté, par commodité, d'utiliser les deux manuscrits du Vatican, accessibles en ligne, dont le texte est suffisamment correct, en particulier celui de Vat. ebr. 289. Le premier, datable au milieu du XIV ${ }^{\mathrm{e}}$ siècle, est un recueil composite, ce commentaire, liturgique, étant suivi de celui, biblique, de Rachi sur les « cinq rouleaux », puis d'abrégés anonymes de De caelo et De generatione et corruptione d'Aristote. Le second, dont les filigranes sont datables entre 1344 et 1354, contient un ensemble de textes de Juda Romano : ce commentaire, puis celui de l'CEuvre de la création, ensuite le Sefer ben porat, enfin la traduction du Liber de causis. Passant par-dessus une introduction en prose d'art obscure, diffuse et au total de peu d'intérêt (f. 1-2 de ce manuscrit), nous avons lu, traduit et commenté les paragraphes («chapitres », mais courts) 1 à 19 (f. $2-7 \mathrm{v}$ ) sur un ensemble de 209 : les chap. 1 à 4 ont un caractère général : division des deux textes, explication de leurs noms et explicitation du rapport mutuel qu'ils impliquent (chap. 4 : le Qaddiš signifiant une propriété qui peut être partagée et atteinte par l'intellect de l'homme qui dépend du temps; la Qedušah, une propriété absolue et inaccessible); les chap. 5-18, à propos du seul premier mot du Qaddiš, à savoir «que sa grandeur soit proclamée », définissent la grandeur divine : début de l'explication; la notion d'infinité essentielle qui n'appartient qu'à Dieu, ne pouvant d'ailleurs exister d'infinité quantitative, ni continue, ni discrète (chap. 7); rapport entre la proclamation initiale de la grandeur de Dieu et celles, qui suivront, de ses autres perfections (chap. 9); encore sur la nature de la grandeur divine et cinq ou six différentes explications qu'on donne de son incommensurabilité; à partir du chap. 19 débute le commentaire de la formule suivante, celle de la sanctification du nom divin, qui sera l'objet de l'étude de l'année prochaine; Juda Romano s'applique d'abord à montrer comment, en tant qu'expression du caractère séparé de l'essence divine, elle découle directement de l'affirmation initiale de la grandeur divine.

Revue des études juives, 162, 2003, p. 195-197), qui souligne l'origine obscure de cette prière, attestée seulement à partir du Moyen Âge mais que des témoignages indirects font remonter à l'Antiquité tardive, aussi bien que la fluidité de son formulaire et son hybridité quant au genre de texte et à la langue dont il use. 